\title{
Non-additive Effects of Structural Factors in Pyridine Catalyzed Reactions of Phenyloxirane with $N$-aroylbenzenesulfonamides
}

\author{
(1D) Igor Shpan'ko, 1,* (1) Irina Sadovaya²
}

\author{
1 Department of Chemistry, Biology and Biotechnology, Vasil’ Stus Donetsk National University, 21 600-richchia Str., Vinnytsia 21021, Ukraine \\ 2 Department of Chemistry, Donetsk National University, 24 Universitetskaya Str., Donetsk 83001, Ukraine \\ * Corresponding author's e-mail address: shpanko16@ukr.net \\ RECEIVED: July 6, 2020 * REVISED: August 18, 2020 * ACCEPTED: August 30, 2020
}

\begin{abstract}
The combined influence of structural factors ( $\mathrm{Y}, \mathrm{Z}$ substituents) on the rate of catalyzed by Z-substituted pyridines reactions of phenyloxirane with $\mathrm{Y}$-substituted $\mathrm{N}$-aroylbenzenesulfonamides in acetonitrile at $293 \mathrm{~K}$ was studied. A cross-correlation analysis of the results of a multifactor kinetic experiment was carried out. A polylinear regression, that adequately describes the non-additive effects of substituents $Y$ and $Z$, was calculated. Due to the interaction of the joint effects of structure, the cross-reaction series exhibits isoparametric properties. The mechanism of the catalytic process has been discussed.
\end{abstract}

Keywords: phenyloxirane, $\mathrm{N}$-aroylbenzenesulfonamides, pyridines, catalysis, cross-correlation analysis, isoparametricity, reaction mechanism.

\section{INTRODUCTION}

0 XIRANES have been studied by experimental and theoretical methods for many decades. Oxirane chemistry is still of current interest to researchers (see, e.g., recent publications $\left.{ }^{[1-5]}\right)$. Of particular interest are oxirane ring opening reactions in which various internal and external factors are varied simultaneously. To describe these reactions, it is necessary to use the multiparameter relationships, based on the polylinearity principle. ${ }^{[6]}$ Polylinear equations contain cross terms, considering interaction (non-additivity) of the effects of mutually varied factors. Interaction of the effects of two varied factors determines such an intriguing property of the polylinear equations as isoparametricity. This term means that the coefficient of sensitivity to the effect of one of factors in one-parameter correlation becomes zero for the isoparametric value of another factor called the isoparametric point. On passing through the isoparametric point, the inversion of the sign of the corresponding sensitivity coefficient takes place (isoparametricity paradox). As a real phenomenon, isoparametricity is a challenge to traditional concepts in chemistry. The isoparametric points are often experimentally inaccessible since they fall in the region of far extrapolation. This may explain the absence of active interest to investigation of the mysterious isoparametricity phenomenon in various fields of natural sciences.

In reactions of aryloxiranes with arenesulfonic and arenecarboxylic acids the interactions structure-structure and structure-temperature types were clearly manifested, ${ }^{[7-10]}$ which made it possible to obtain convincing experimental evidence of the isoparametricity phenomenon. Of particular importance is the proof of the physical reality of such an aspect of isoparametricity as the widely discussed enthalpy-entropy compensation effect. ${ }^{[8-10]}$

Our recent study ${ }^{[11]}$ showed that in the catalyzed by Z-substituted pyridines reaction of phenyloxirane with such representative of $\mathrm{NH}$ acids as $\mathrm{N}$-benzoylbenzenesulfonamide, there was no interaction between the effects of substituents $Z$ and temperature. This reaction turned out to be isoenthalpic with respect to variation of substituents $Z$ $\left(\Delta H_{Z}^{\ddagger}=\right.$ const, $\left.\delta_{Z} \Delta H^{\ddagger}=0\right)$. The effects of substituents $Z$ were manifested exclusively due to variation of the activation entropy $\left(\delta_{z} \Delta G^{\ddagger}=-T \delta_{z} \Delta S^{\ddagger}\right)$. It is of interest to elucidate the

(cc) $\mathbf{B Y}$ This work is licensed under a Creative Commons Attribution 4.0 International License. 
joint effects of the structures of the oxirane substrate, acidic reagent, and catalyst in such reactions.

In this work, we have studied the combined influence of substituents $Y$ and $Z$ on the rate of catalyzed by pyridines Z-Py 3a-3d reactions of phenyloxirane $\mathbf{1}$ with $\mathrm{N}$-aroylbenzenesulfonamides $\mathbf{2 a - 2 c}$ in acetonitrile at $293 \mathrm{~K}$ (Scheme 1). The results of a multifactor kinetic experiment were subjected to cross-correlation analysis, the isoparametric properties of the cross-reaction series were examined, the mechanism of the catalytic process was discussed.

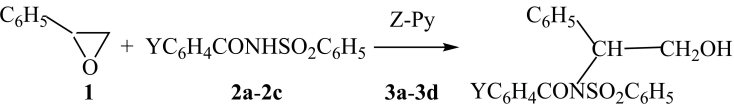

$$
\begin{aligned}
& \mathrm{Y}=4-\mathrm{Me} \text { (2a), } \mathrm{H}(\mathbf{2 b}), 4-\mathrm{NO}_{2} \text { (2c) } \\
& \mathrm{Z}=4 \text {-Et (3a), H (3b), 3-COOEt (3c), 3-CN (3d) }
\end{aligned}
$$

Scheme 1 . Reactions of oxirane 1 with imides 2 catalyzed by pyridines 3 .

\section{EXPERIMENTAL}

Chemically pure grade acetonitrile was dried and distilled over $\mathrm{P}_{2} \mathrm{O}_{5}$ and then over $\mathrm{CaH}_{2}$. Commercial phenyloxirane 1 (Merck, content of the main substance no less than 98\%) and chemically pure grade pyridines $\mathbf{3 a - 3} \mathbf{d}$ were vacuumdistilled. Acidic reagents $\mathbf{2 a - 2 c}$ (mixed imides of benzenesulfonic and arenecarboxylic acids) were synthesized and purified as described in work. ${ }^{[12]}$ The reaction products (Scheme 1) were primary alcohols, 2-( $N$-aroyl- $N$-benzenesulfonyl)amino-2-phenylethanols. ${ }^{[13]}$

The reaction rate was measured as a decrease in the amount of acids $\mathbf{2}$, as was described in our earlier work. ${ }^{[14]}$ The kinetics of the reactions were studied at more than tenfold excess of the oxirane substrate (S) with respect to initial concentrations of the acidic reagent $(\mathrm{NH})$ : $[\mathrm{S}]_{0}>>$ $[\mathrm{NH}]_{0}=0.477-1.91 \mathrm{~mol} \mathrm{dm}^{-3}$; in turn, the concentration of pyridines ranged within $m=0.0116-0.0860 \mathrm{~mol} \mathrm{dm}^{-3}$. Under those conditions, the reactions had a common third order: the first order for each of the reagents and the catalyst, so that the rate of the process at the constant concentration of the catalyst $m$ was described by the following equation: $-d[\mathrm{NH}] / d t=k_{1}[\mathrm{NH}]=k_{3}[\mathrm{~S}]_{0}[\mathrm{NH}] m$. The observed pseudo-first order rate constants $k_{1}\left(\mathrm{~s}^{-1}\right)$ were constant up to $70-80 \%$ conversion of the acidic reagent (the error in measuring $k_{1}$ was $\leq 5 \%$ ). The second-order rate constants $k_{2}\left(\mathrm{~mol}^{-1} \mathrm{dm}^{3} \mathrm{~s}^{-1}\right)$ were obtained as $k_{2}=k_{1} /[\mathrm{S}]_{0}$. Numerical values of the effective catalytic third-order rate constants $k_{\mathrm{yz}}\left(\mathrm{mol}^{-2} \mathrm{dm}^{6} \mathrm{~s}^{-1}\right)$ were calculated from four kinetic runs at different concentrations $m$ using the $k_{2}=k_{\mathrm{yz}} m$ linear equation (correlation coefficient $r \geq 0.998$ ).
The accuracy of determining the kinetic and correlation parameters was estimated in term of the standard deviation $S$, which was determined by a statistical method from $n$ experimental data points. Statistical data processing was carried out for the confidence level of 0.95.

\section{RESULT AND DISCUSSION}

The values of the rate constants $k_{\mathrm{yz}}$ for the catalytic reactions (Scheme 1 ) are listed in Table 1. For a quantitative assessment of the effects of the substituents $Y$ and $Z$ on the rate the following Hammett-type equations were used:

$$
\begin{aligned}
& \log k_{Y Z}=\log k_{H Z}+\rho_{Y}{ }^{Z} \sigma_{Y} \\
& \log k_{Y Z}=\log k_{Y H}+\rho_{Z}^{Y} \sigma_{Z}
\end{aligned}
$$

Eq. (1) describes reactions with variable substituents $Y$ and fixed substituents $Z$, and Eq. (2) describes the effects of variable substituents $Z$ at fixed substituents $Y$. The Figure below shows examples of linear dependences according to Eq. (2).

The results of processing kinetic data (Table 1 ) using Eqs. (1) and (2) are given in Table 2. They show that when passing from one fixed substituent $Z(Y)$ to another, the sensitivity coefficients $\rho_{Y}^{z}\left(\rho_{Z}^{Y}\right)$ noticeably change. This indicates the interaction of the effects of the substituents $Y$ and $Z$, which is also confirmed by the statistically significant values of the cross-interaction coefficient, which is equal to the slopes of the dependences (3) and (4).

$$
\begin{aligned}
& \rho_{Y}{ }^{\mathrm{Z}}=(1.20 \pm 0.01)+(0.47 \pm 0.03) \sigma_{\mathrm{Z}} \\
& S=0.016, r=0.999, n=4 . \\
& \rho_{\mathrm{Z}}{ }^{\mathrm{Y}}=(-0.89 \pm 0.03)+(0.47 \pm 0.07) \sigma_{\mathrm{Y}} \\
& S=0.050, r=0.989, n=3 .
\end{aligned}
$$

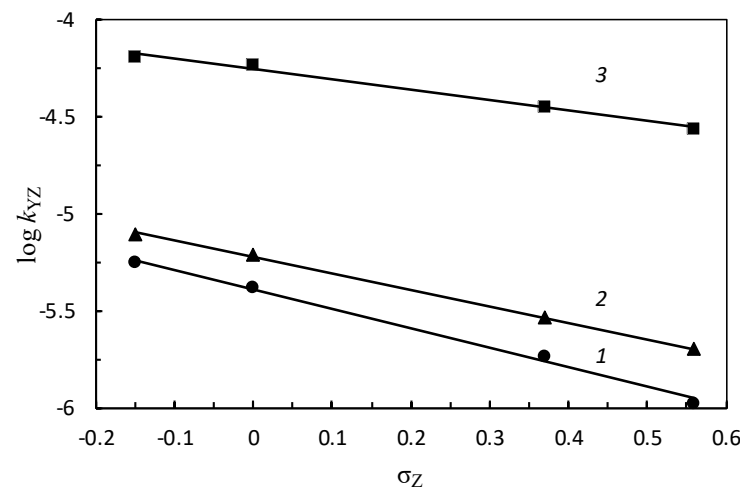

Figure 1 . The effects of substituents $Z$ on catalytic activity of pyridines $3 a-3 d$ in reactions of oxirane 1 with imides $2 a(1)$, $2 b(2), 2 c(3)$ in acetonitrile at $293 \mathrm{~K}$. 
Table 1. Rate constants $k_{y z}$ for catalyzed by pyridines 3a-3d reactions of phenyloxirane 1 with imides $2 a-c$ in acetonitrile at $293 \mathrm{~K}$

\begin{tabular}{ccccc}
\hline \multirow{5}{*}{ Imides } & \multicolumn{4}{c}{$10^{5} \mathrm{krz}_{\left(\mathrm{mol}^{-2} \mathrm{dm}^{6} \mathrm{~s}^{-1}\right)}$ Pyridines } \\
\cline { 2 - 5 } & 3a & $3 b$ & $3 c$ & $3 d$ \\
\hline $2 a$ & $0.56 \pm 0.02$ & $0.42 \pm 0.01$ & $0.185 \pm 0.008$ & $0.106 \pm 0.005$ \\
$2 b$ & $0.79 \pm 0.01$ & $0.617 \pm 0.005$ & $0.292 \pm 0.007$ & $0.20 \pm 0.01$ \\
$2 c$ & $6.47 \pm 0.07$ & $5.80 \pm 0.02$ & $3.55 \pm 0.03$ & $2.74 \pm 0.06$ \\
\hline
\end{tabular}

Table 2. The coefficients of Eq. (1) and Eq. (2) for catalyzed by Z-substituted pyridines 3a-3d reactions of phenyloxirane 1 with Y-substituted imides $2 a-2 c$ in acetonitrile at $293 \mathrm{~K}$

\begin{tabular}{|c|c|c|c|c|}
\hline Fixed substituent Z $\left(\sigma_{z}\right)$ & $\log k_{\mathrm{HZ}}$ & $\rho r^{2}$ & $r$ & $S$ \\
\hline 4-Et (-0.15) & $-5.08 \pm 0.02$ & $1.13 \pm 0.04$ & 0.999 & 0.030 \\
\hline$H(0)$ & $-5.19 \pm .0 .02$ & $1.21 \pm 0.04$ & 0.999 & 0.026 \\
\hline 3-COOEt (0.37) & $-5.51 \pm 0.01$ & $1.36 \pm 0.03$ & 0.999 & 0.022 \\
\hline 3-CN (0.56) & $-5.71 \pm 0.01$ & $1.48 \pm 0.02$ & 0.999 & 0.013 \\
\hline Fixed substituent $Y\left(\sigma_{Y}\right)$ & $\log k_{Y H}$ & $\rho z^{Y}$ & $r$ & $S$ \\
\hline $4-\mathrm{CH}_{3}(-0.17)$ & $-5.39 \pm 0.02$ & $-1.00 \pm 0.05$ & 0.998 & 0.027 \\
\hline$H(0)$ & $-5.22 \pm 0.01$ & $-0.85 \pm 0.02$ & 0.999 & 0.010 \\
\hline $4-\mathrm{NO}_{2}(0.78)$ & $-4.25 \pm 0.01$ & $-0.53 \pm 0.03$ & 0.997 & 0.016 \\
\hline
\end{tabular}

To quantify the joint effects of substituents $Y$ and $Z$ on the rate of the catalytic process, we used Eq. (5) applicable to the two-parameter variant of polylinearity principle. ${ }^{[6]}$

$$
\log k_{Y Z}=\log k_{H H}+\rho_{Y}{ }^{Z=H} \sigma_{Y}+\rho_{Z}^{Y=H} \sigma_{Z}+\rho_{Y Z} \sigma_{Y} \sigma_{Z} .
$$

Here, $k_{\mathrm{HH}}$ is the rate constant of the standard reaction $\left(Y=Z=H, \sigma_{Y}=\sigma_{Z}=0\right), \rho_{Y}{ }^{Z=H}$ and $\rho_{Z}^{Y=H}$ are the sensitivity coefficients of standard reaction series, and $\rho_{\mathrm{yz}}$ is the above-mentioned cross-interaction coefficient. By processing the results of the multifactor kinetic experiment (Table 1) using Eq. (5), we obtained the polylinear regression, Eq (6) ( $R$ is the multiple correlation coefficient, $F$ is the Fisher test).

$$
\begin{aligned}
& \log k_{Y Z}=(-5.20 \pm 0.01)+(1.20 \pm 0.02) \sigma_{Y}+ \\
& (-0.89 \pm 0.03) \sigma_{Z}+(0.47 \pm 0.05) \sigma_{Y} \sigma_{Z} \\
& S=0.023, R=0.999, F=2675, n=12 .
\end{aligned}
$$

Regression [Eq. (6)] describes the catalytic reactions to a high degree of accuracy, as is evidenced by its statistical characteristics and the correspondence of the value of cross-interaction coefficient $\rho_{\mathrm{yz}}$ to its values in Eqs. (3) and (4). Due to the statistical significance of cross-interaction coefficient ( $\rho_{Y z}=0.47 \pm 0.05$ ), regression, Eq. (6), exhibits the isoparametric properties. Its attributes are isoparametric points with respect to the constants of the substituents $Y$ $\left(\sigma_{Y}{ }^{I P}=-\rho_{Z}^{Y=H} \rho_{Y Z}{ }^{-1}=1.89\right)$ and $Z\left(\sigma_{Z}^{I P}=-\rho_{Y}^{Z=H} \rho_{Y Z}{ }^{-1}=-2.55\right)$, as well as the same value of the rate constant $k_{y z}$ at these points $\left(\log k_{\mathrm{YZ}}{ }^{\mathrm{IP}}=\log k_{\mathrm{HH}}-\rho_{\mathrm{Y}}^{\mathrm{Z}=\mathrm{H}} \rho_{\mathrm{Z}}{ }^{\mathrm{Y}=\mathrm{H}} \rho_{\mathrm{Yz}}{ }^{-1}=-2.93\right)$. The experimental achievement of the isoparametric point $\sigma_{Z}{ }^{I P}$, at which the reaction rate should not depend on substituents $Y$ in imides 2, is not possible due to the deficit of electrondonor substituents $Z$ with $\sigma_{z}=-2.55$. The isoparametric point $\sigma_{Y} \mathbb{P}^{P}$, where the changes in the structure of substituents $Z$ in pyridines $\mathbf{3}$ should not affect the reaction rate, also is experimentally unattainable.

The fulfilment of the cross correlations of the kinetic data [Eqs. (3), (4) and (6)] for the studied reactions (Scheme 1 ) is indicative of a uniform interaction between the effects of substituents $Z$ in pyridines $\mathbf{3}$ and substituents $Y$ in imides $\mathbf{2}$ within the framework of a single mechanism of the catalytic process (Scheme 2). The catalytic role of pyridines is to enhance the nucleophilic properties of the acidic reagent $\mathrm{Y}-\mathrm{NH}$ (imides 2 ) due to the acid-base interaction in the first equilibrium stage. Among the possible ionic intermediates formed in an acetonitrile medium, imide anion $\mathrm{Y}^{-} \mathrm{N}^{-}$and pyridinium cation $\mathrm{HPy}^{+}-\mathrm{Z}$ should possess the strongest catalytic effect. In the second equilibrium stage, complex $\mathbf{A}$ is formed with a hydrogen bond between oxirane 1 and the pyridinium cation $\mathrm{HPy}^{+}-\mathrm{Z}$. The oxirane substrate activated in this way undergoes a nucleophilic attack by the imide anion $\mathrm{Y}-\mathrm{N}^{-}$with the formation of transition state $\mathbf{B}$ in the third rate-determining stage. This stage proceeds according to the mechanism 
$A_{N} D_{N}$ with electrophilic assistance from the pyridinium cation $\mathrm{HPy}^{+}-\mathrm{Z}$ to the $\mathrm{C}-\mathrm{O}$ bond cleavage in the oxirane ring. In view of the above considerations, the mechanism of the catalytic action of pyridines presented in Scheme 2 can be characterized as basic with electrophilic assistance.

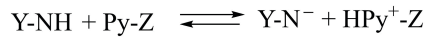

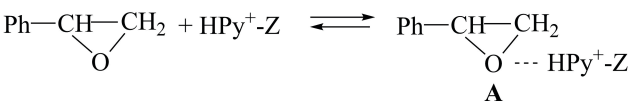

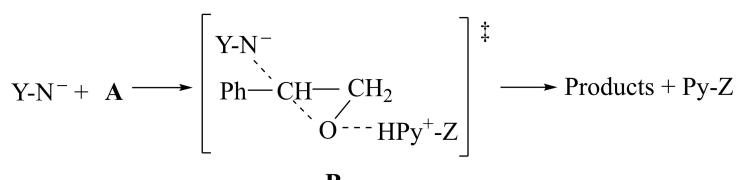

Scheme 2 . The catalytic action of pyridines 3 in the reactions of oxirane 1 with imides 2 .

According to Scheme 2, in the multistage catalytic process the interaction of the effects of substituents $Y$ and $Z$ can occur both in the first stage of the acid-base interaction and in the third stage of the oxirane ring opening. As for the interaction of structure effects in the first stage, it cannot play a dominant role in the catalytic process, because the opposite signs of the cross-interaction coefficient in the studied reaction system $\left(\rho_{\mathrm{Yz}}=0.47\right)$ and in the process of proton transfer from phenols to pyridines $\left(\rho_{\mathrm{yz}}=-0.57\right){ }^{[15]}$ For this reason, the isoparametric points with respect to structural parameters in these crossreaction series differ significantly in nature. They correspond to the maximum of the acid-base interaction in the studied system $\left(\sigma_{Y}^{\mathbb{P P}}=1.89, \sigma_{Z}^{\mathbb{P}}=-2.55\right)$ and its minimum in the phenols-pyridines system $\left(\sigma_{Y}{ }^{\mathrm{PP}}=-2.36\right.$, $\left.\sigma_{z^{I P}}=1.89\right)$

In view of the above, we can conclude that in the studied cross-reaction series the interaction of the effects of the substituents $Y$ and $Z$ manifests itself much more intensively in the oxirane ring opening stage than in stage of acid-base interaction.

\section{CONCLUSION}

In the presented study we revealed, based on the results a cross-correlation analysis of experimental kinetic data (Table 1), the interaction (non-additivity) of the joint effects of the structural factors ( $Y, Z$ substituents) in catalyzed by pyridines 3 reactions of oxirane 1 with $\mathrm{NH}$-acids 2 (Scheme 1). The influence of the cross-varied factors on the rates of catalytic reactions is adequately described by the polylinear regression [Eq. (6)]. Owing to the interaction of the effects of the structural factors the studied crossreaction series exhibits isoparametric properties, the quantitative criteria of which are the cross-interaction coefficient $\rho_{Y Z}=0.47$, and isoparametric points with respect to the constants of the substituents $Y\left(\sigma_{Y}{ }^{P}=1.89\right)$ and $Z$ $\left(\sigma_{Z} I^{P}=-2.55\right)$. In accordance with the properties of the isoparametric dependences at the isoparametric point $\sigma_{Y}{ }_{Y}^{\mathbb{P}}\left(\sigma_{Z}{ }_{Z}\right)$ the rate of catalytic reactions should not depend on substituent $Z(Y)$. Both isoparametric points are experimentally unattainable. The positive sign of the crossinteraction coefficient $\rho_{\mathrm{Yz}}$ indicates the dominant role of the interaction of structural effects in the rate limiting stage of the oxirane ring opening (Scheme 2).

Acknowledgment. The study was carried out within the Fundamental Research Programme funded by the Ministry of Education and Science of Ukraine (Project No. 0116U002519).

Conflict of interests. The authors have declared no conflict of interest.

\section{REFERENCES}

[1] Zh. Chen, S. M. Nasr, M. Kazemi, M. Mohammadi, Mini-Revs Org. Chem. 2020, 17, 352-362. https://doi.org/10.2174/1570193X16666190723111746

[2] K. Yutilova, Yu. Bespal'ko, E. Shved, Croat. Chem. Acta. 2019, 92, 357-367.

https://doi.org/10.5562/cca3505

[3] D. T. Smith, J. T. Njardarson, Top. Heterocycl. Chem. 2016, 41, 281. https://doi.org/10.1007/7081_2015_147

[4] V. Gilanizadeh, B. Zeynizadeh, Curr. Chem. Lett. 2015, 4, 153-158.

https://doi.org/10.5267/j.ccl.2015.6.002

[5] G. S. Singh, K. Mollet, M. D'Hooghe, N. De Kimpe, Chem. Rev. 2013, 113, 1441-1498.

https://doi.org/10.1021/cr3003455

[6] V. A. Palm, Osnovy kolichestvennoi teorii organicheskikh reaktsii [Fundamentals of the quantitative theory of organic reactions], Khimiya, Leningrad, 1977, pp. 35-54, 260-276, 310-314.

[7] I. V. Shpan'ko, I. V. Sadovaya, N. V. Kulikova, Russ. J. Org. Chem. 2011, 47, 687-693.

https://doi.org/10.1134/S107042801105006X

[8] I. V. Shpan'ko, I. V. Sadovaya, Kinet. Catal. 2014, 55, 56-63. https://doi.org/10.1134/S002315841401011X

[9] I. V. Shpan'ko, I. V. Sadovaya, Reac. Kinet. Mech. Cat. 2018, 123, 473-484.

https://doi.org/10.1007/s11144-017-1340-6

[10] I. V. Shpan'ko, I. V. Sadovaya, ARKIVOC 2019, 6, 93-104. https://doi.org/10.24820/ark.5550190.p010.924

[11] I. V. Shpan'ko, I. V. Sadovaya, Russ. J. Gen. Chem. 2019, 89, 2358-2363.

https://doi.org/10.1134/\$1070363219120053 
[12] G. I. Derkach, G. F. Dregval', A.V. Kirsanov, Zh. Obshch. Khim. 1960, 30, 3402-3407.

[13] I. V. Shpan'ko, I. V. Sadovaya, Ukr. Khim. Zh. 2015, 81, 124-127.
[14] I. V. Shpan'ko, I. V. Sadovaya, A. M. Kitaigorodskii, Ukr. Khim. Zh. 2003, 69, 111-115.

[15] D. Clotman, J. P. Muller, Th. Zeegers-Huyskens, Bull. Soc. Chim. Belg. 1970, 79, 689-697.

https://doi.org/10.1002/bscb.19700791112 